\title{
$\angle S$ Research Square \\ Impact of Region- of-interest Size and Location on Quantitative Contrast-enhanced Ultrasound of Canine Splenic Perfusion
}

\section{Simona Morabito}

Universita degli Studi di Messina

\section{Simona Di Pietro}

Universita degli Studi di Messina

Luca Cicero ( $\nabla$ cicero.luca@libero.it ) Istituto Zooprofilattico Sperimentale della Sicilia "A. Mirri", Via Gino Marinuzzi 3, 90100 Palermo, Italy https://orcid.org/0000-0003-4386-7723

\section{Annastella Falcone}

Universita degli Studi di Messina

\section{Luigi Liotta}

Universita degli Studi di Messina

\section{Rosalia Crupi}

Universita degli Studi di Messina

\section{Giovanni Cassata}

Istituto Zooprofilattico Sperimentale della Sicilia Adelmo Mirri

\section{Francesco Macrì}

Universita degli Studi di Messina

\section{Research article}

Keywords: Contrast-enhanced ultrasonography, Dog, Region of interest, Spleen, Sonovue, Qontrast

Posted Date: August 24th, 2020

DOI: https://doi.org/10.21203/rs.3.rs-59709/v1

License: (1) This work is licensed under a Creative Commons Attribution 4.0 International License. Read Full License

Version of Record: A version of this preprint was published at BMC Veterinary Research on August 11th, 2021. See the published version at https://doi.org/10.1186/s12917-021-02973-z. 


\section{Abstract}

\section{Background}

During the contrast enhanced ultrasound (CEUS) the features of regions of interest (ROI) can affects the value of the perfusion parameters obtained from a time intensity curves (TIC). In veterinary medicine, the influence of ROI size and location during the CEUS exam has been described using the kidney as target organ, with contrasting results. No guidelines have been reported for the ideal placement or size of the ROI during the quantitative analysis of CEUS in canine spleen.

The aim of this study was to evaluate the effect of the size and location of ROls in the spleen of conscious dogs on perfusion variables determined using contrast-enhanced ultrasonography with sulphur hexafluoride.

\section{Results}

A prospective, observational study on 15 adult client-owned mixed-breed dogs was performed using a system equipped with contrast-tuned imaging technology. Qualitative and quantitative assessment of the enhancement pattern of the spleen were performed. Three square ROls $\left(0.05 \mathrm{~cm}^{2}\right)$ were manually drawn in a row and spaced $1 \mathrm{~mm}$ apart, placing adjacent ROls at three different depths. Three medium rectangular ROIs $\left(0.3 \mathrm{~cm}^{2}\right)$ encompass the 3 smaller ROls of each row and a single large square ROI ( 1 $\mathrm{cm}^{2}$ ) containing all the previous ROls were drawn. Software analysis of generated time-intensity curves within each ROI allowed to calculate the perfusion parameters: peak enhancement, time to peak, regional blood flow and volume and mean transit time.

The coefficient of variation for all parameters was always lowest in the largest ROI in comparison with the other smaller ROIs. The ROI A and B, positioned in proximal and middle levels respectively, showed coefficients of variation similar to the largest ROI. The analysis of variance model showed a significant effect of location and size of ROls in the quantitative perfusion analysis of the canine spleen, with a reduction of perfusion parameters in the distal $\mathrm{ROI}$.

\section{Conclusions}

The analysis of splenic perfusion through quantitative evaluation of enhancement with a contrast agent into a large enough and proximal to the ultrasound beam area of splenic parenchyma may be of clinical importance in the diagnosis of splenic disorders.

\section{Background}

The contrast enhanced ultrasound (CEUS) is an imaging technique useful to obtain a reliable quantification of tissue perfusion. It is performed by injecting into the bloodstream the gas-filled microbubbles, stabilized by an outer shell, that cause an enhancement of the microvasculature, providing realtime assessment of tissue perfusion [1]. Second generation ultrasound contrast agents not diffuse in the 
extra-vascular space, reflecting only the tissue vasculature [2]. The change of brightness over time is a function of the contrast agent inflow and outflow in a selected region of interest (ROI). Data obtained with this technique need to be processed with a dedicated software that allows to analyze signals of the blood-pool contrast agent without background disturbances. The brightness and its distribution inside a $\mathrm{ROI}$ is analyzed to describe typical enhancement patterns for a tissue or a lesion compared to the adjacent tissue and to obtain the perfusion variables, calculated from time-intensity curves (TIC) in a ROI [3].

The standardization of TIC analysis is crucial to obtain a reliable quantitative CEUS evaluation and clinical decision-making.

Ex vivo and in vivo studies reported that the ROI can affects the value of the perfusion parameters obtained from a TIC [3-6]. The ROI was drawn and located manually by the operator, the size and localization being therefore variable.

In veterinary medicine, the influence of ROI size and location during the CEUS exam has been described, using the kidney as target organ, on experimental models[4,5,7] and on anaesthetized or conscious dogs[8-10]. There are some conflicting results regarding the ideal method for selecting the ROI in quantitative analysis of renal perfusion using CEUS. Leinonen et al. (2011) found that there was a significant inverse association between the size of the ROI and the peak intensity [3]. Other authors recommended the use of the largest possible ROI to minimize the influence of renal perfusion heterogeneity $[11,12]$. Conversely, drawing three smaller ROIs inside the large ROI, the heterogeneity of diffusion of the contrast medium in the area was eliminated, proving the option to use small or large ROls during renal CEUS in dogs [10].

The vascular patterns of the normal or pathological spleen in healthy dogs and cats has been described using the CEUS analysis and recent studies have been focused on canine normal splenic perfusion patterns and blood pool phase peculiarities of splenic lesions, with the purpose of improve the diagnostic procedures in discriminating a benign from a malignant tumor, and in monitoring therapy [13-21]. However, no guidelines have been reported for the ideal placement or size of the ROI during the quantitative analysis of CEUS in canine spleen, in order to assess the effect of the ROI's variables on perfusion parameters of the splenic tissue.

The aim of this study is to evaluate the effect of the ROI size and location on perfusion parameters in the spleen of not-sedated dogs, based on the hypothesis that the ROl's variables would significantly affect the mean value of canine splenic perfusion variables.

\section{Results}

In all dogs included in the study B-Mode examination showed a normal echo-pattern of the splenic tissue. No focal lesions were identified, and the splenic parenchyma showed a diffusely homogeneous echo 
pattern. Colour Doppler detected a normal vasculature and excluded the presence of ischemic vascular lesions or intravascular thrombi.

Contrast-enhanced ultrasound showed a perfusion of the splenic tissue characterized by an early wash-in phase with a rapid enhancement of the small splenic arteries (10 $\pm 2 \mathrm{sec}$.), a heterogeneous phase of enhancement of the spleen that became homogeneous at the peak enhancement ( $45 \pm 15 \mathrm{sec}$.) and a slow decrease of enhancement in the wash-out phase (Figure 2). The absence of splenic lesions was confirmed.

Post-processing quantitative analysis clarified the variations of the signal intensity induced by the passage of the microbubbles and created parametric maps of the regions of interest under investigation, through the processing of the signal of the wash-in and wash-out curves.

The coefficient of variation for all perfusion parameters was always lowest in the largest ROI (ROI MAX) in comparison with the other smaller ROIs. The coefficient of variation for peak enhancement in ROI MAX were 0.18 , whereas other perfusion parameters were equal or higher than 0.2 .

The coefficient of variation for all parameters of the ROI A and B, located in proximal and middle levels respectively, showed values similar to those of the ROI MAX, whereas the coefficient of variation for parameters of the distal ROI C deviated from those of the ROI MAX, resulting generally higher. The data are shown in Table 1.

The mean values \pm standard deviation (SD) of each perfusion parameters in the examined ROIs, together with their statistical significance, are shown in Table 2.

The application of the analysis of variance (ANOVA) model showed a significant effect of location and size of ROls in the quantitative perfusion analysis of the canine spleen.

In particular, a significant decrease of the mean values for all measured parameters of ROI C compared to $\mathrm{ROI} A$ and ROI B was observed. Furthermore, the comparison among the medium-sized ROls with the largest ROI MAX showed that statistically differences there were only with distal ROI C.

No adverse reactions during the procedure occurred.

\section{Discussion}

In veterinary medicine, CEUS has become a reference method in imaging, thanks to its remarkable safety in use, lack of ionizing radiation or toxicity and low costs [22].

It is known that factors related to technical variables and contrast medium or patient-related factors contribute to variability in the quantitative analysis of CEUS and in the resulting perfusion parameters [20]. 
Technique related factors (use of the three-way stopcock, calibre of catheter, contrast medium injection speed and volume of the saline flush) have to be standardised to reduce their influence on time to peak values $[9,23]$. The size of the organ and the characteristics of the parenchyma could also influence the acoustic "strength" of the beam and determine variations in the peak intensity [24].

In this study the procedures were standardized as much as possible, attempting to minimize the influence of the handling of the bubbles on the quantitative imaging analysis. All scanner parameters were fixed: a low mechanical index was chosen to minimize microbubble disruption and allow their accumulation in the microvasculature and allowing the same expert operators to perform the procedure every time, as suggested to avoid variability in clinical applications of CEUS $[18,25]$. Time gain compensation and overall gain were decreased before the contrast agent injection, in order to suppress most background tissue signals.

Efforts were made to minimize patient related factors that can significantly influenced the peak enhancement and the mean transit time. The dogs enrolled in the study on basis of the ultrasound characteristics of the splenic parenchyma, which was homogenous in all subjects; moreover, the spleen is a superficial intra-abdominal organ, whose position is little influenced by the physical conformation of the animal.

Anaesthetic drugs can also modify the quantitative analysis of the contrast medium due to their pharmacokinetic and metabolism by the lung, primarily when inhalers are used $[15,20]$. Intravenous anaesthetics alter the CEUS quantitative analysis, modifying the blood pressure and heart rate: the peak intensity time seems to be much faster in awake than anesthetized dogs with propofol [26]. Conversely, butorphanol did not affect the cardiovascular parameters during the evaluation of feline renal perfusion with CEUS $[15,21,27]$.

In our setting the effect on splenic blood flow by anesthetic or sedative drugs can be ruled out, since the dogs were awake.

In this study the location and size of ROI were analysed, in order to detect their effect on the perfusion parameters variability. Our results showed that a deviation for measuring values at different depths was present. If a ROI in an area of subjectively homogenous parenchyma is moved vertically there are deviations for the resulting data, with a largest coefficient of variation at the deepest ROI. The deviations differ for the different parameters in a range between $18 \%$ and $42 \%$ with a mean deviation of $27 \%$. Peak enhancement seem to be the most stable parameter for depth positioning variations.

Furthermore, the lowest coefficient of variation for all perfusion parameters has been found when a single large square ROI $\left(1 \mathrm{~cm}^{2}\right)$ was drawn on the splenic parenchyma, in comparison of the other smaller ROls encompassed in the previous one. Although there was again a tendency for certain parameters, as peak enhancement, to be more

stable than others, all parameters had a coefficient of variation less than $40 \%$. 
This findings underline that during the CEUS of canine spleen time intensity curves should not be analysed in a deep level of the splenic parenchyma, while would be appropriate drawn a ROI as large as possible, considering the most reliable values of perfusion parameters obtained by time intensity curve.

Furthermore, when comparing more than one ROI, e.g. in a tumour vs. representative parenchyma, would be appropriate compare them in the same depth.

The ANOVA analysis allowed us to better understand the observed variability of perfusion parameters: they were significantly greater in the proximal ROIs compared to the distal ROls. A reason to explain this finding is that the ultrasound beam could be attenuated in the deeper areas.

Furthermore, the perfusion parameters appear to be independent from ROI size on condition that they are positioned at the proximal and middle levels of the splenic parenchyma.

\section{Conclusions}

The analysis of splenic perfusion through quantitative evaluation of enhancement with a contrast agent into a large enough and proximal to the ultrasound beam area of splenic parenchyma may be of clinical importance in the diagnosis of splenic disorders. Results of the present study could be useful in defining guidelines for the selection of ROIs and controlling variability in the use of CEUS to evaluate splenic perfusion.

\section{Methods}

\section{General materials}

Signed informed dogs' owners consent about methods and purposes of this study were obtained. The protocol was approved by the Animal Ethics Board of the Department of Veterinary Sciences, University of Messina (protocol number: 13/2017). All treatments, housing and animal care followed the EU Directive 2010/63/EU on the protection of animals used for scientific purposes.

Fifteen mixed-breed dogs, 8 males and 7 females, were enrolled in this study. All dogs were presented at the Veterinary Teaching Hospital of the University of Messina between October 2017 and February 2019 for the assessment of renal perfusion through CEUS, due to a mildly increase of blood urea and creatinine. Age of the enrolled dogs ranged from 1 to 7 years and the mean body weight was $30.9 \pm 3.6$ (SD) kg.

Dogs were included in the study only if the spleen was homogenous at ultrasonography. Dogs with focal or multifocal large lesions were excluded and fifteen dogs have been selected. The enrolled dogs, submitted to a clinical examination, had normal heart rate, blood pressure, capillary refill time, respiratory rate and hydration status.

\section{Ultrasonography procedure}


All the dogs were subjected to B-mode ultrasonography, Doppler ultrasonography and CEUS. Ultrasound and Doppler examination were performed by the same investigator (FM) using a scanner Mindray M9 (Shenzhen, China), equipped with a linear probe (10-12-MHz). The dogs were not sedated and were manually restrained in the right lateral recumbency, the hair was clipped, alcohol (70\%) and coupling gel were applied to the skin.

Spleen tissue was considered normal if the margins were regular and smooth and the parenchyma showed a finely textured and homogeneous pattern, more echogenic than liver and the cortex of left kidney. Colour Doppler was performed to evaluate the splenic vascularization and rule out the presence of splenic intravascular thrombotic structures or ischemic lesions.

CEUS examination was performed, using a linear transducer $(10-12-\mathrm{MHz})$ probe with contrast agent

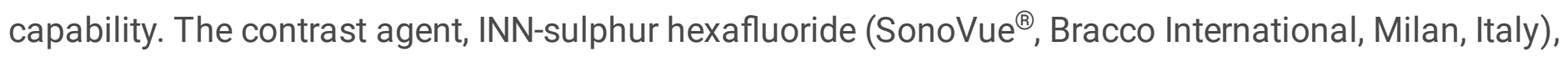
was prepared following the manufacturer's recommendations and was rapidly injected $(0.05 \mathrm{~mL} / \mathrm{kg}$ of body weight) via a three-way stopcock and 18-gauge catheter placed in a cephalic vein, in accordance with a methodology previously reported $[28,29]$. Each dog received two bolus injections of contrast agent, which were standardised and administered by the same investigator (SDP). The first bolus was administered to assess the kidney while the second bolus, injected approximately 5 to 10 minutes after the first bolus, was used for the assessment of the spleen. Injection of the contrast agent and activation of a timer were started simultaneously and video clips were recorded for 2 minutes. As animal were not sedated, care was taken to keep the probe in the same position for at least 2 minutes. The spleen was examined with a mechanical index set at a low value (0.09). Standardised settings were adopted for time gain compensation, overall gain and focal zone, as previously reported [19]

Raw data (good-quality video clips) obtained during CEUS were stored digitally on a hard disk. A trained investigator (LL) analysed all the functional data. A qualified operator (SM) drowned a total of 13 quadrangular or rectangular ROIs. The smallest ROls $\left(0.05 \mathrm{~cm}^{2}\right)$ were numbered in sequential numerical order from 1 to 9 . They were drawn in groups of three, at a distance of one millimetre, on three different depth levels: proximal, middle and distal. Around each group of three ROIs, a larger ROI $\left(0.3 \mathrm{~cm}^{2}\right)$ was drawn, indicated with the letters A, B and C. The total of the 12 ROls were grouped into a higher ROI (1 $\mathrm{cm}^{2}$ ), named as ROI MAX (Figure 1). The use of image-analysis software produced post-processing analysis (Qontrast ${ }^{\circledR}$, Bracco Imaging, Milan, Italy). This software processed the raw data allowing measurement of tissue perfusion in ROls and determining variables automatically. A time-intensity curve, which is a parametric curve of time versus signal intensity (SI), was also generated for each ROI. The maximal SI was defined as a white band in the grey scale bar (8 bit). The software generated the following parameters: peak enhancement, time to peak (TTP), mean transit time (MTT),regional blood volume (RBV) and regional blood flow (RBF) for each ROI. These parameters were defined as follows:

- peak enhancement as the percentage increase in SI (from 0 to 100 as maximal intensity) reached during transit of the contrast agent at a specific time point; 
- TTP as the interval until the maximum SI of the contrast agent;

- MTT as the circulation time of the contrast agent in the examined tissue;

- RBF as the ratio between RBV and MTT.

\section{Statistical analysis}

Descriptive statistical analysis of the quantitative CEUS-derived parameters revealed a normal distribution for each value. To evaluate statistical differences among the bodyweight of the enrolled dogs a paired Student t-test was used.

To define the influence of the location and size of the ROI on perfusion parameters, the coefficient of variation was calculated for each of them. If a parameter showed the lowest coefficient of variation, it was considered acceptable and reliable.

Data obtained from the quantitative CEUS-derived parameters were analysed by ANOVA with the GLM procedure (SAS V.9.4, SAS Institute, 2001), using ROI size and location as variables. All data were expressed as mean \pm standard deviation (SD). Statistical significance was assessed at the $\mathrm{P}<0.05$ level.

\section{Abbreviation}

CEUS: Contrast-enhanced ultrasonography; MTT: Mean transit time; RBF: Regional blood flow; RBV:

Regional blood volume; ROI: Region of interest; SI: Signal Intensity; TIC: Time-intensity curve; TTP: Time to peak.

\section{Declarations}

\section{Ethics approval and consent to participate}

The protocol was approved by the Animal Ethics Board of the Department of Veterinary

Sciences, University of Messina.

The dog owners were informed about the methods and purpose of the study and gave their written informed consent.

\section{Consent for publication}

Written informed consent was obtained from the dogs' owners for publication of this manuscript.

\section{Availability of data and materials}


The datasets used and/or analysed during the current study are available from the corresponding author on reasonable request.

\section{Funding}

There were no funding resources for this study.

\section{Competing interests}

The authors declare that they have no competing interests.

\section{Authors' contribution}

FM, GC and SDP conceived the study. FM and SM performed the diagnostic

examinations. FM, SDP and LL performed the CEUS off-line quantification analysis,

statistical analysis and wrote the manuscript. LC and AF managed the patients and contrast

media during the ultrasounds and contributed to reviewing the manuscript. RC, LC and AF analysed the data and assisted to drafting the manuscript. All authors read and approved the final manuscript.

\section{Acknowledgments}

No third-party funding or support was received in connection with this study or the writing or publication of the manuscript.

\section{References}

1. Haers $\mathrm{H}$, Saunders JH. Review of clinical characteristics and applications of contrast-enhanced ultrasonography in dogs. J Am Vet Med Assoc. 2009;234:460-470.

2. Doury M, Dizeux A, de Cesare A, Lucidarme O, Pellot-Barakat C, Bridal SL, Frouin F. Quantification of tumor perfusion using dynamic contrast-enhanced ultrasound: impact of mathematical modeling. Phys Med Biol. 2017;62:1113-1125.

3. Leinonen MR, Raekallio MR, Vainio OM, et al. The effect of the sample size and location on contrast ultrasound measurement of perfusion parameters. Vet Radiol Ultrasound 2011;52:82-87.

4. Claudon M, Barnewolt CE, Taylor GA, Dunning PS, Gobet R, Ba- dawy AB. Renal blood flow in pigs: changes depicted with contrast-enhanced harmonic US imaging during acute urinary obstruction. Radiology 1999;212:725-731.

5. Taylor GA, Barnewolt CE, Claudon M, Dunning PS. Depiction of renal perfusion defects with contrastenhanced harmonic sonography in a porcine model. Am J Roentgenol 1999;173:757-760. 
6. Mule S, De Cesare A, Lucidarme O, Frouin F, Herment A. Regularized estimation of contrast agent attenuation to improve the imaging of microbubbles in small animal studies. Ultrasound Med Biol 2008;34: 938-948.

7. Dong Y, Wang W, Cao J, et al. Quantitative evaluation of contrast-enhanced ultrasonography in the diagnosis of chronic ischemic renal disease in a dog model. PLOS ONE 2013;8:e70337.

8. Haers H, Smets P, Pey P, et al. Contrast harmonic ultrasound appearance of consecutive percutaneous renal biopsies in dogs. Vet Radiol Ultrasound 2011;52:640-647)

9. Waller KR, O’Brien RT, Zagzebski JA. Quantitative contrast ultrasound analysis of renal perfusion in normal dogs. Vet Radiol Ultrasound 2007;48:373-377.

10. Macrì F, Di Pietro S, Liotta L, et al. Effects of size and location of regions of interest examined by use of contrast-enhanced ultrasonography on renal perfusion variables of dogs. Am J Vet Res. 2016;77:869-876.

11. Wei K, Le E, Bin JP, et al. Quantification of renal blood flow with contrast- enhanced ultrasound. JACC 2001;37:1135-1140.

12. Schneider AG, Calzavacca P, Schelleman A, et al. Contrast-enhanced ultrasound evaluation of renal microcirculation in sheep. Intensive Care Med Exp 2014;2:33.

13. Ohlerth S, O’Brien RT. Contrast ultrasound: general principles and veterinary clinical applications. Veterinary Journal, 2007;174:501-512.

14. Nakamura K, Sasaki N, Yoshikawa M, et al. Quantitative contrast-enhanced ultrasonography of canine spleen. Veterinary Radiology \& Ultrasound, 2009;50:104-108.

15. Rossi F, Fina C, Stock E, et al. Effect Of Sedation On Contrast-Enhanced Ultrasonography Of The Spleen In Healthy Dogs. Vet Radiol Ultrasound 2016 May;57(3):276-81

16. Rossi F, Leone VF, Vignoli M, et al. Use of contrast-enhanced ultrasound for characterization of focal splenic lesions. Vet Radiol Ultrasound 2008;49:154-164.

17. Ohlerth S, Dennler M, Ruefli E, et al. Contrast harmonic imaging characterization of canine splenic lesions. J Vet Intern Med 2008;22:1095-1102.

18. Ivancic M, Long F, Seiler GS. Contrast harmonic ultrasonography of splenic masses and associated liver nodules in dogs. J Am Vet Med Assoc 2009;234:88-94.

19. Mangano C, Macri F, Di Petro S, et al. Use of contrast-enhanced ultrasound for assessment of nodular lymphoid hyperplasia (NLH) in canine spleen. BMC Vet Res. 2019;11;15:196. Doi: 10.1186/s12917-019-1942-5.

20. Tang MX, Mulvana H, Gauthier T, et al. Quantitative contrast-enhanced ultrasound imaging: a review of sources of variability. Interface Focus, 2011;1:520-539.

21. Leinonen MR, Raekallio MR, Vainio OM, et al. Effect of anesthesia on contrast-enhanced ultrasound of the feline spleen. Vet $J$ 2011;190:273-277.

22. Seiler GS, Brown JC, Reetz JA, et al. Safety of contrast-enhanced ultrasonography in dogs and cats: 488 cases (2002-2011). J Am Vet Med Assoc. 2013;242:1255-1259. 
23. Talu E, Powell RL, Longo ML et al. Needle size and injection rate impact microbubble contrast agent population. Ultrasound Med. 2008;34,1182 - 1185.

24. Bargellini P, Orlandi R, Paloni $C$, et al. Contrast-enhanced ultrasonographic characteristics of adrenal glands in dogs with pituitary-dependent hyperadrenocorticism Vet Radiol Ultrasound, 2013;54:283292.

25. Mullin L, Gessner R, Kwan J, et al. An in vivo evaluation of the effects of anesthesia carrier gases on ultrasound contrast agent circulation. Ultrasonics Symp. (IUS) 2009;6:126-131.

26. Nyman, H.T., Kristensen AT, Kjelgaard-Hansen M et.al. Contrast-enhanced ultrasonography in normal canine liver. Evaluation of imaging and safety parameters, Vet Radiol Ultrasound 2005:46:243-250.

27. Stock E, Vanderperren K, Van der Vekens E, Haers H, Duchateau L, Polis I, Hesta M, Saunders JH.The effect of anesthesia with propofol and sedation with butorphanol on quantitative contrast-enhanced ultrasonography of the healthy feline kidney. Vet J. 2014;202(3):637-639.

28. Quartuccio M., Mangano C., Macrì F., et al. Contrast-enhanced ultrasound evaluation of testicular interstitial cell tumours in conscious non-sedated dogs. Veterinarni Medicina, 2018, 63: 125-130.

29. Macrì F, Di Pietro S, Mangano C, et al. Quantitative evaluation of canine urinary bladder transitional cell carcinoma using contrast-enhanced ultrasonography. BMC Vet Res. 2018; 14:84

\section{Tables}

Table 1: Average values $( \pm S D)$ of the perfusion variables of the largest (ROI MAX) and medium-size ROIs, from the most superficial (ROI A) to the deepest (ROI C).

\begin{tabular}{|lllll|}
\hline Variable & ROI MAX & ROI A $(1,2,3)$ & ROI B $(4,5,6)$ & ROI C $(7,8,9)$ \\
\hline Peak (\%) & $34 \pm 8.1^{\mathrm{a}}$ & $35 \pm 7.9^{\mathrm{a}}$ & $35 \pm 7.2^{\mathrm{a}}$ & $32 \pm 8.1^{\mathrm{b}}$ \\
\hline TTP (s) & $46 \pm 10.2^{\mathrm{a}}$ & $46 \pm 15.4^{\mathrm{a}}$ & $47 \pm 12.5^{\mathrm{a}}$ & $44 \pm 13.5^{\mathrm{b}}$ \\
\hline RBF (L/min) & $43 \pm 11.2^{\mathrm{a}}$ & $46 \pm 11.9^{\mathrm{a}}$ & $44 \pm 10.9^{\mathrm{a}}$ & $41 \pm 11.1^{\mathrm{b}}$ \\
\hline MTT (s) & $77 \pm 17.5^{\mathrm{a}}$ & $79 \pm 24.2^{\mathrm{a}}$ & $78 \pm 19.1^{\mathrm{a}}$ & $72 \pm 17.6^{\mathrm{b}}$ \\
\hline RBV & $3425 \pm 1309.6^{\mathrm{a}}$ & $3546 \pm 1311.5^{\mathrm{a}}$ & $3550 \pm 1407.2^{\mathrm{a}}$ & $3074 \pm 1320.4^{\mathrm{b}}$ \\
\hline
\end{tabular}

a, b Means in the same row with different superscript differ $(P<0.05)$

Table 2: Coefficients of variation concerning parameters and different variables, values expressed in \%. 


\begin{tabular}{|lllll|}
\hline & ROI proximal (A) & ROI middle (B) & ROI distal (C) & ROI Max \\
\hline PEAK & 19.46 & 18.43 & 21.58 & 17.89 \\
\hline TTP & 24.36 & 25.59 & 30.51 & 22.12 \\
\hline RBV & 36.49 & 38.67 & 41.68 & 36.94 \\
\hline RBF & 23.92 & 23.66 & 24.16 & 20.76 \\
\hline MTT & 29.23 & 24.48 & 25.35 & 21.68 \\
\hline
\end{tabular}

$\mathrm{ROI}=$ region of interest; Peak= peak enhancement; TTP=time to peak; RBV=red blood volume; RBF=red blood flow; MTT=mean transit time

\section{Figures}
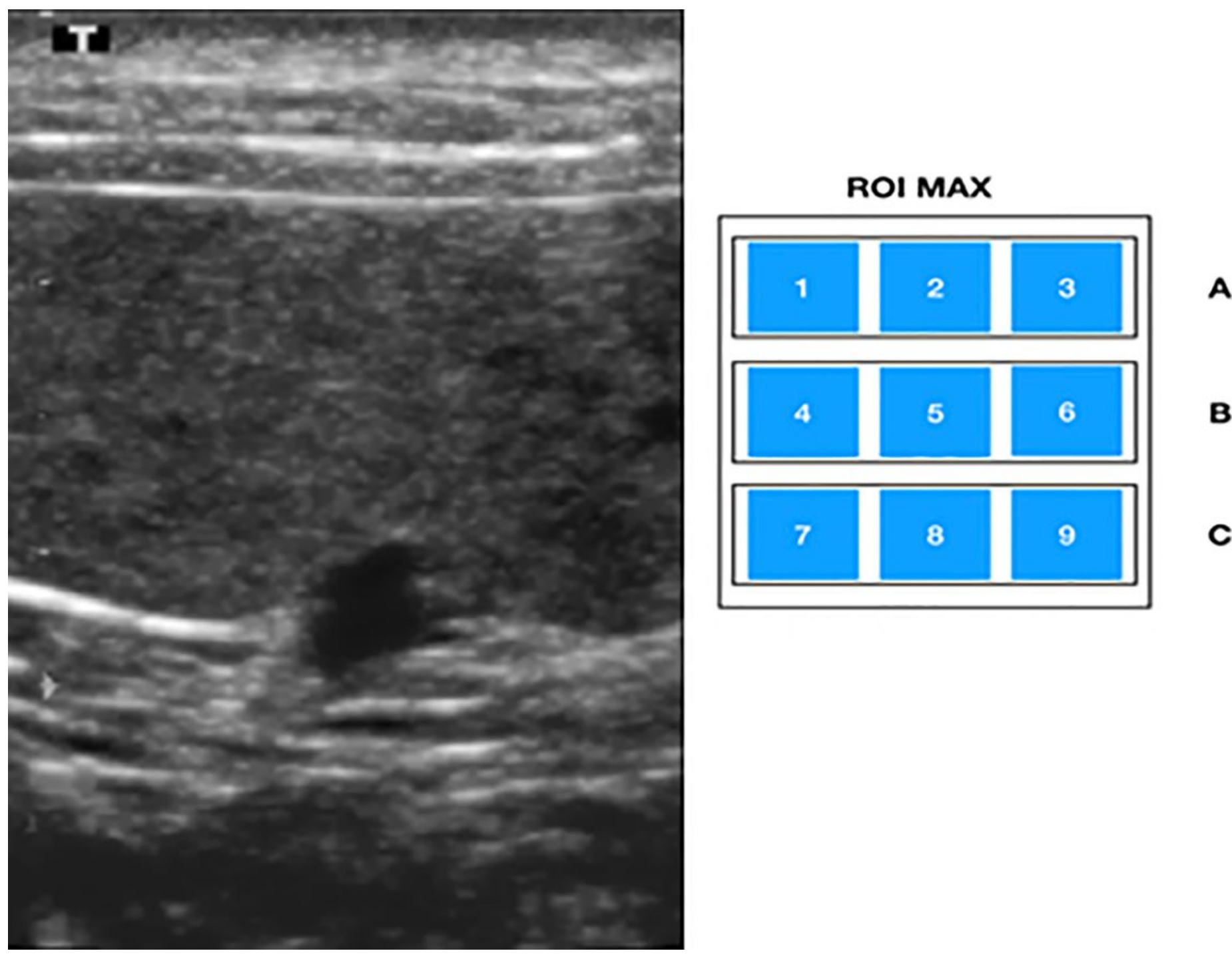

Figure 1 
Schematic representation of the ROls used in the evaluation of the splenic parenchyma. Nine square ROIs (1-9) each measuring $0.05 \mathrm{~cm} 2$ are separated by $0.1 \mathrm{~cm}$. Three larger rectangular ROls (A-C) have an area of $0.3 \mathrm{~cm} 2$, and each encloses a row of three smaller ROIs. A single large square ROI encompasses all others with an area of $1 \mathrm{~cm} 2$.
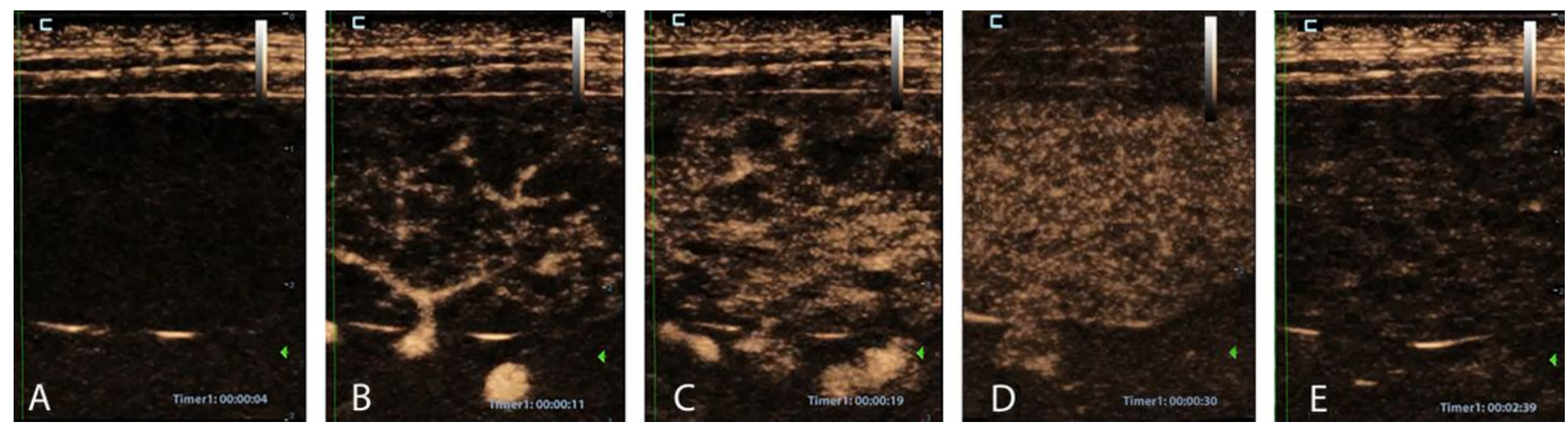

\section{Figure 2}

Representative images of the vascularization pattern in the spleen of a dog during the CEUS study. A: precontrast image after the bolus administration of contrast medium. B-E: post-contrast injection images over time. 